\title{
EL DEBATE EN TORNO AL SEGURO DE SALUD PÚBLICO Y PRIVADO EN ESPAÑA: DESDE LA TRANSICIÓN POLÍTICA A LA LEY GENERAL DE SANIDAD (1975-1986)
}

The debate regarding public and private health insurance in Spain: From the political transition to the General Law of Health (1975-1986)

\author{
MARGARITA VILAR-RODRÍGUEZ \\ Universidad de A Coruña \\ mvilar@udc.es \\ JERÒNIA PONS-PONS \\ Universidad de Sevilla \\ ipons@us.es
}

Cómo citar/Citation

Vilar-Rodríguez, M. y Pons-Pons J. (2018).

El debate en torno al seguro de salud público y privado en España:

desde la transición política a la Ley General de Sanidad (1975-1986).

Historia y Política, 39, 261-290.

doi: https://doi.org/10.18042/hp.39.10

(Recepción: 09/01/2017. Evaluación: 08/03/2017. Aceptación: 26/06/2017. Publicación: 17/04/2018)

\section{Resumen}

Este trabajo persigue como principal objetivo analizar cómo se gestaron y se debatieron en el Parlamento las bases del actual modelo sanitario español desde el inicio de la transición democrática hasta la aprobación de la Ley General de Sanidad en 1986. Durante este período se produjo un importante debate sobre el modelo y funcionamiento del sistema sanitario en Espańa que orbitaba en torno al papel que debía representar el Estado en la gestión, financiación y prestación de servicios y su 
competencia o cooperación con el sector privado. El estudio pone en evidencia que el debate sanitario ha venido protagonizado por dos modelos diferentes de sanidad que se han enfrentado desde los primeros momentos de la transición hasta el presente. La herida entre estas dos facciones sigue abierta y el consenso global lejos de ser alcanzado.

\title{
Palabras clave
}

Sanidad; modelo sanitario; España; transición política.

\begin{abstract}
This paper aims to analyze how the foundations of the current Spanish health model were developed and debated in Parliament from the beginning of the democratic transition until the approval of the General Health Law in 1986. During this period there was an important political debate on the model of the health system in Spain that orbited around the role that the State should represent in the management, financing and provision of services and its competence or cooperation with the private sector. The study shows that the health debate has been carried out by two different models of health that have been faced from the first moments of the transition to the present. The wound between these two factions remains open and the global consensus far from being reached.
\end{abstract}

\section{Keywords}

Health; health model; Spain; political transition. 


\section{SUMARIO}

I. INTRODUCCIÓN. II. EL SEGURO DE SALUD EN LA TRANSICIÓN DEMOCRÁTICA: ENTRE LAS HERENCIAS Y LAS REFORMAS (1975-1978). III. EL DEBATE SOBRE EL MODELO SANITARIO EN LOS PRIMEROS PASOS DE LA DEMOCRACIA (1979-1981). IV. LA APROBACIÓN DE LA ANSIADA LEY DE SANIDAD (1986): DEBATE Y CONSECUENCIAS: 1. El debate sobre la Ley General de Sanidad. 2. El modelo establecido por la Ley General de Sanidad. V. CONCLUSIONES: LA AGENDA PENDIENTE TREINTA AÑOS DESPUÉS. BIBLIOGRAFÍA.

\section{INTRODUCCIÓN}

La muerte de Franco el 20 de noviembre de 1975 abrió la puerta hacia la transición de España a la democracia, un proceso finalmente exitoso que tuvo mucho de incoherente e improvisado ${ }^{1}$. La mayoría de historiadores, políticos o periodistas han caracterizado a la transición española como un proceso singular por tres razones fundamentales: primero, porque fue una reforma política confeccionada desde la legalidad anterior — sin ruptura global-; segundo, porque consiguió un nuevo consenso histórico en el país - aunque no ausente de controversia y de confrontación política a corto y medio plazoque derivó en la aprobación de la Constitución de 1978; tercero, porque cristalizó en un Estado democrático y autonómico, estable y plural, que consiguió integrarse en Europa en apenas una década.

En conjunto, la década de 1970 resultó un período complicado para España, pues se sucedieron crisis ocasionales, algunas más graves que otras, en el ámbito político (la incertidumbre y la complejidad de la transición), económico (las conocidas como crisis del petróleo que asolaron, sobre todo, a los países capitalistas occidentales) y social (oleada de manifestaciones en un marco de efervescencia política). Este difícil contexto se vio reflejado en la composición de los gobiernos y principales cargos políticos de la época: de un lado, muestran una imagen de continuidad más que de ruptura; de otro, revelan las dificultades para administrar las grandes demandas sociales del momento y encajar a la vez las diversas voluntades políticas en un país donde el estado de bienestar estaba por desarrollar.

1 Sobre estas cuestiones, véanse García Delgado et al. (2008); Gallego (2008), y Carr (2009). 
La cobertura sanitaria se posicionaba como una de las principales demandas de la población espańola durante la transición. La aprobación del Seguro Obligatorio de Enfermedad en 1942 convirtió la sanidad en una pieza clave del discurso propagandístico paternalista de «justicia social» utilizado por la dictadura franquista para amortiguar las tensiones internas del país en un marco de control de la población, represión laboral y pésimas condiciones de $v$ ida $^{2}$. Sin embargo, el modelo sanitario establecido por la dictadura revelaba un sistema imperfecto y con graves problemas, ahogado por un déficit financiero crónico y con capacidad de cobertura muy limitada tanto en términos de prestaciones ofrecidas como en porcentaje de población cubierta. Además, el Estado franquista se desentendió por completo de su financiación y el sistema permaneció costeado básicamente por empresarios y trabajadores, quienes destinaban un porcentaje de su raquítico salario a las cotizaciones y recibían unas prestaciones irrisorias, cada vez más depreciadas por la fuerte inflación del período ${ }^{3}$. En consecuencia, los trabajadores se convirtieron en las principales víctimas de las imperfecciones del sistema sanitario vigente durante la dictadura. Este modelo protector careció además de dos pilares básicos en los modelos de cobertura social vigentes en los países de la Europa occidental tras la Segunda Guerra Mundial: la universalización de las prestaciones y el carácter redistributivo desde el punto de vista social ${ }^{4}$. Sin una reforma fiscal que aportase el sostén financiero y bajo las limitaciones propias de un régimen dictatorial resultaba imposible avanzar en la modernización del sistema sanitario.

Los años setenta suponen una década de dos esperas históricas en el ámbito sanitario: la llegada de la democracia y la creación del Ministerio de Sanidad ${ }^{5}$. Se confiaba en que ambos aspectos permitiesen establecer en España un sistema sanitario público de corte europeo. En este objetivo se concentraron las expectativas de políticos, profesionales médicos y de la sociedad en

2 Estos aspectos han sido ampliamente estudiados en Pons y Vilar (2014).

3 Los salarios reales se mantuvieron bajos en la posguerra en un marco de falta de libertades y derechos laborales, véase Vilar (2004).

4 Véanse Comín (1996; 2010). En particular, como señala Comín (2015: 174), «la dictadura de Franco fue una excepción en la Europa occidental, lo que explica que la política económica y fiscal de Franco fuese una reliquia del pasado, utilizada en los países fascistas, que contrastó con las aplicadas en la Europa democrática e incluso en otras dictaduras como Portugal». La rigidez en la recaudación del Estado durante la dictadura (basada en impuestos indirectos y con un elevado fraude y ocultación) limitó los gastos y obligó a recurrir de manera abusiva a las emisiones de deuda pública.

5 Francia (1997) y Guillén (2000). 
general. Pero el proceso no resultó sencillo. Primero, porque el sector sanitario vivió durante estos años un permanente conflicto en muchos frentes, pues no existía acuerdo entre las diferentes fuerzas políticas, sociales y profesionales sobre el modelo sanitario. Segundo, porque la reforma sanitaria necesitaba de la reforma previa del marco hacendístico y político, que no se consolidó hasta la reforma tributaria de 1977 y la aprobación de la Constitución en 1978. Tercero, porque casi cuarenta años de dictadura habían dejado como herencia una estructura sanitaria llena de problemas organizativos y financieros y de deficiencias difíciles de resolver de manera inmediata.

En conjunto, desde el inicio de la transición democrática hasta la aprobación de la Ley General de Sanidad en 1986, se produjo un importante debate sobre el modelo y funcionamiento del sistema sanitario español ${ }^{6}$. Este proceso, apenas estudiado en la historiografía, resultó clave para definir el modelo sanitario vigente en España en las décadas posteriores. Este trabajo tiene como principal objetivo aportar un análisis pormenorizado de este debate. El estudio pone en evidencia que el debate sanitario ha venido protagonizado por dos modelos diferentes de sanidad que se han enfrentado desde los primeros momentos de la transición hasta el presente. La herida entre estas dos facciones sigue abierta y el consenso global, lejos de ser alcanzado. Partiendo de estas ideas, resulta de gran interés estudiar cómo se gestaron y se debatieron en el Parlamento las bases del actual sistema sanitario espańol, un país que ha logrado alcanzar las mejores posiciones en los rankings internacionales tanto en indicadores de salud de sus ciudadanos como en valoración de su sistema sanitario ${ }^{7}$.

\section{EL SEGURO DE SALUD EN LA TRANSICIÓN DEMOCRÁTICA: ENTRE LAS HERENCIAS Y LAS REFORMAS (1975-1978)}

El Instituto Nacional de Previsión (INP), centro del poder sanitario durante el franquismo y brazo ejecutor de la política social durante la

6 Las formas de cobertura de la asistencia sanitaria se han vinculado a los modelos estatales de bienestar establecidos por Esping-Andersen (1990; 1996). A partir de aquí, otros autores han aportado matices y diferencias entre países, incluso entre aquellos que inicialmente parecían formar parte de la misma categoría. Sobre los modelos de bienestar en el sur de Europa en perspectiva comparada, véanse Ferrera (1996) y Guillén (2006).

7 Los estudios disponibles sobre estos indicadores muestran la buena posición española en este ámbito, véase, por ejemplo, Medeiros y Schwierz (2015). 
dictadura, se convirtió en centro principal de las críticas de los que perseguían una renovación profunda en la sanidad con el cambio de régimen ${ }^{8}$. De un lado, el INP se identificaba demasiado con el modelo de política social de la dictadura como para poder revestirlo de un traje democrático y convertirlo en abanderado de la reforma sanitaria en la transición. De otro, el INP había mostrado su incapacidad para resolver los problemas de financiación acumulados y había fracasado en la europeización de un sistema sanitario diseñado en un marco dictatorial y con enormes fallas en términos de cobertura y gestión ${ }^{9}$. Por este y otros motivos, el INP estaba condenado a desaparecer antes o después en un contexto de creciente conflicto sanitario agravado por los efectos negativos derivados de la crisis económica. El profesor Camilo Lluch afirmaba en un artículo de prensa publicado en 1976 que «la Seguridad Social en España está inmersa en una profunda crisis que afecta a su financiación, a sus gastos, a su gestión y a su control $»^{10}$. Tomando como base este diagnóstico, proponía un replanteamiento completo del modelo que debería contener necesariamente un control democrático del presupuesto de la Seguridad Social y un nuevo modelo de financiación que corrigiese los negativos efectos redistributivos sobre la renta del esquema aplicado hasta entonces, basado en cargos a los propios trabajadores, bien mediante cuotas sobre salarios, bien mediante la elevación de los precios de los bienes y servicios que consumen para compensar la cuota empresarial. Según reflejan las Memorias Estadisticas de la Seguridad Social, en 1970 el 90,26 \% de los ingresos del Sistema de la Seguridad Social procedía de las cuotas pagadas por empresarios y trabajadores; el casi $10 \%$ restante se repartía entre las subvenciones del Estado y otros recursos minoritarios. En 1975 la situación apenas había variado; las cuotas de empresarios y trabajadores mantenían un porcentaje del $88,62 \%$. Dentro de las cotizaciones sociales, las cuotas al seguro de enfermedad representaban en torno al $50 \%$ del importe total. Este esquema era insostenible. Sin embargo, una reforma fiscal previa parecía imprescindible (aunque no suficiente) para una mayor financiación del sistema por parte del Estado. Pero el país tenía muchos deberes pendientes.

En mayo de 1977, el diario Ya recogía las líneas principales de los programas sanitarios de tres partidos políticos donde quedaban claros los diferentes modelos perseguidos ${ }^{11}$. El Partido Demócrata Popular (más tarde en

8 Jurado (1993): 18.

9 Sobre estos aspectos, véase Pons y Vilar (2014).

10 «La seguridad social en la encrucijada», Ya, 17-11-1976, Hemeroteca Archivo Linz, R-58860.

11 Para este párrafo, véase «La reforma sanitaria, a debate», $Y a$, 14-5-1977, Hemeroteca Archivo Linz, R-59715. 
AP) defendía «la sanidad como un servicio público esencial que debe encauzar el estado; regionalización de las redes de hospitales y demás servicios dirigida a lograr una cobertura total de las necesidades asistenciales; y libre elección de médico». Por su parte, la Federación Social Demócrata (más tarde UCD) perseguía «la creación del servicio de salud pública que beneficie al conjunto de la población española, con máxima descentralización de servicios y posibilidad de libre elección de médico y centro de asistencia mediante el pago de una cantidad mínima proporcional a su coste y renta». Finalmente, el Partido Socialista Popular (más tarde en PSOE) proponía «la implantación de una política de salud integral, servicio nacional de salud, único, público y gratuito, financiado exclusivamente a través de impuestos y compatible durante el período de adaptación de la misma con la práctica privada de la medicina; paralelo impulso de campañas de prevención y educación sanitaria».

Hubo que esperar al resultado derivado de las primeras elecciones democráticas de junio de 1977 para abordar el conflicto. Tras la apertura del período constituyente, llegaron los primeros intentos de reforma. Los principales escaños en el Congreso se repartían entre UCD (165), PSOE (118), PCE (20) y AP/PP (16) ${ }^{12}$. Dentro de este marco, un PSOE con claras aspiraciones de alcanzar el Gobierno ejerció una dura y activa oposición, también en el ámbito de la sanidad. Enrique Sánchez de León ${ }^{13}$ se convirtió en ministro de Sanidad (julio 1977-marzo 1979) durante el primer gobierno de la UCD elegido por las urnas y liderado por Adolfo Suárez. Su ministerio se creó sobre dos organizaciones estatales: la Dirección General de Sanidad, hasta ahora bajo el paraguas del Ministerio de Gobernación, y la Seguridad Social, hasta entonces bajo la competencia del Ministerio de Trabajo ${ }^{14}$. Con esta unificación se ponían las bases para el nacimiento del Ministerio de Sanidad y Seguridad Social, como respuesta a la necesidad de unificar en un solo organismo estatal

12 Datos recogidos de Linz et al. (2005), cuadro 14.26.

13 Licenciado en derecho, ingresó en el Cuerpo Superior de Inspectores de Trabajo y Seguridad Social del Ministerio de Trabajo en 1960. En 1971 pasó a formar parte del Congreso como procurador general del Estado. Entre otros cargos, había sido nombrado director general de Ordenación de la Seguridad Social en el primer Gobierno de la monarquía. Información obtenida del Archivo del Congreso de los Diputados.

14 El primer Ministerio de Sanidad en la historia de Espańa se había creado durante la II República, siendo su titular Francisco Largo Caballero. El ministerio desapareció tras la Guerra Civil y sus competencias fueron asumidas por el Ministerio de la Gobernación hasta 1977. 
las dispersas competencias que en materia de salud pública se distribuían hasta entonces en ministerios diversos ${ }^{15}$.

El sector más crítico con la sanidad vigente calificó este proceso como una oportunidad perdida para reformar de raíz y modernizar la sanidad espańola, puesto que «el control del ministerio quedó en manos del grupo de presión más continuista, defensor del modelo sanitario elaborado por el INP» ${ }^{16}$. A pesar de las críticas, el ministro siguió adelante estableciendo dos principales objetivos a cumplir durante su mandato: la reforma de la Seguridad Social y la elaboración de una ley de sanidad ${ }^{17}$. La primera perseguiría los objetivos de simplificación y racionalización económica y de democratización de los órganos de vigilancia y control para obtener la máxima rentabilidad social. La segunda debía clarificar las responsabilidades públicas y privadas así como los objetivos prioritarios del modelo sanitario del país.

En el mes de octubre de 1977 comenzó el estudio para la elaboración de una ley de salud ${ }^{18}$. El 1 de diciembre de 1977 se celebra el debate de la primera sesión informativa de la Comisión de Sanidad y Seguridad Social en el Congreso de los Diputados ${ }^{19}$. En su discurso de apertura, el ministro Sánchez de León presentó los principales problemas de la sanidad española y las líneas maestras a seguir por su ministerio, que «nace compuesto de retazos y con muchos problemas enquistados $»^{20}$. Entre los problemas destacó tres muy graves que afectaban a los pilares del sistema. Primero, reconoció la regresividad de la seguridad social en España puesto que las bases teóricas de cotización más próximas a la remuneración real se mostraban en los salarios más bajos y no en los más altos, donde realmente existía la mayor capacidad contributiva ${ }^{21}$. Segundo, asumió la persistencia de una gestión sanitaria obsoleta que «vinculaba la higiene, la pobreza, la caridad, la asistencia, la medicina preventiva en una marańa de organismos y actuaciones dignas de ser corregidas ${ }^{22}$. Tercero, puso énfasis en el excesivo gasto en farmacia (89 mil millones de pesetas al año), que convertía a España en el séptimo país del mundo en consumo farmacéutico, y la ineficiente distribución del personal sanitario (por

\footnotetext{
Decreto 1558/77 de 4 de julio, BOE 05/07/1977.

Francia (1997): 123-126.

«Los reformadores», $A B C, 22-1-1978$, p. 3.

18 «Estudio de la Reforma de asistencia sanitaria y de las bases de la futura ley de la salud", $A B C, 02-10-1977$.

19 Diario de Sesiones del Congreso de los Diputados, año 1977, núm. 32, p. 1159.

20 "Hoy, nueva sesión de la cumbre de la Moncloa», $A B C, 18-12-1977$.

21 Diario de Sesiones del Congreso de los Diputados, año 1977, núm. 32, p. 1173.

22 Diario de Sesiones del Congreso de los Diputados, año 1977, núm. 32, p. 1183.
} 
aquel entonces sesenta mil médicos, 3400 odontólogos, dieciocho mil farmacéuticos, siete mil veterinarios, ochenta mil ayudantes técnicos sanitarios y cinco mil matronas). Estos problemas urgían una reforma sanitaria que permitiese conseguir una mayor operatividad, con una reducción del gasto y la progresiva clarificación económica y financiera, y corregir los graves defectos del sistema de prestaciones que gozaba de clara impopularidad, sobre todo en el caso de la asistencia primaria.

¿Pero qué modelo sanitario proponía la UCD en 1977? El ministro Sánchez León parecía tenerlo claro: «La Seguridad Social necesita democratizarse y socializarse, pero no estatalizarse ${ }^{23}$. Es decir, se proponía un sistema sostenido en la liberalización de la primera asistencia, la interrelación entre las distintas instituciones, la asistencia psiquiátrica, el impulso de la medicina de familia y la descentralización. Teniendo en cuenta estos aspectos, el Ministerio presentó un anteproyecto de ley de sanidad en diciembre de 1977. El texto generó duras críticas desde el Colegio de Médicos, los sindicatos y los partidos de la oposición. Los diputados del PSOE Guillermo Galeote y Vicente Martín ejercieron una dura oposición en torno a dos cuestiones ${ }^{24}$ : el papel del Estado en el ámbito de la sanidad (funciones a asumir y estrategia de la política sanitaria) y la necesidad de generar transparencia y confianza a la sociedad en torno a la «jungla de la Seguridad Social». Dentro de este clima de tensión, el avance del proyecto se enquistó.

Unos meses más tarde, los representantes del Ministerio de Sanidad justificaban el retraso del proyecto argumentando que «en tanto no se apruebe la Constitución por referéndum, lo único que puede hacer el Ministerio es ir arbitrando una serie de normas internas que faciliten el buen funcionamiento de la sanidad», sin avanzar más ${ }^{25}$. Bajo su argumento, entendían que la reforma sanitaria debía esperar hasta que se diese a conocer el modelo socio-económico del país, que debería venir establecido por el texto constitucional. A los problemas de corte político había que sumar la falta de control de los gastos y de los ingresos y la ineficacia en la prestación de servicios, denunciada por médicos y periodistas en la prensa diaria, pues «la actual estructura de ingresos y pagos supone de hecho un campo abonado para el descontrol, las corruptelas y el pago de extratipos a mutualidades y demás organismos autónomos

23 «Sánchez de León: Antes de fin de ańo se presentará a las Cortes el proyecto de Reforma sanitaria", $A B C$, 5-11-1977.

24 Diario de Sesiones del Congreso de los Diputados, año 1977, núm. 32, p. 1185 y ss.

25 "La reforma sanitaria se establecerá tras la aprobación de la Constitución», El País, 30-8-1978 y "El difícil e inalcanzable camino de la reforma sanitaria», El País, 15-9-1978. 
difícilmente perseguible, dado que en estos momentos la Seguridad Social cuenta con más de dos centenares de comisiones gestoras que actúan poco más o menos como reinos de taifas $»^{26}$. A este conflicto había que añadir «el atraso y falta de pago de las cuotas de la Seguridad Social por parte de gran número de empresas» afectadas por la crisis económica de aquellos años, que casi había conducido al INP a la bancarrota en el verano de 1977. Ante los graves desequilibrios financieros, el Ministerio estableció medidas restrictivas sobre algunas partidas de gastos de la Seguridad Social. Las guardias de médicos, las pensiones alimenticias de los enfermos hospitalizados y las adquisiciones de útiles, lencería y material fungible de los hospitales se convirtieron en el principal objetivo de los recortes ${ }^{27}$. El malestar social creció.

Los Pactos de la Moncloa incluían un importante programa de reformas de la Seguridad Social centradas en inyectar transparencia y responsabilidad pública como contraposición al INP, que representaba por aquellos tiempos la opacidad y fracaso del sistema sanitario en España. El problema radicó en que la mayor parte de los acuerdos tomados no se llevaron a la práctica por los sucesivos Gobiernos de la UCD, lo que contribuyó al desencanto social y abrió un período de fuertes debates políticos y mediáticos con dos grupos bien definidos, uno de ruptura con el sistema vigente y otro de integración ${ }^{28}$. Por una parte, la mayoría de la opinión pública, sindicatos, partidos de izquierda (PSOE y PCE) y minoritarios sectores profesionales del ámbito sanitario defendían un sistema basado en un servicio nacional de salud, que integrase las instituciones sanitarias de todas las Administraciones Públicas, de cobertura universal y financiación básicamente estatal. Por otra, la mayoría de los médicos y de la Administración Sanitaria, junto con los partidos de la UCD y AP, abogaban por un sistema de seguros sociales, financiado mayoritariamente a través de la Seguridad Social (con participación marginal del Estado) y gestionado a través de conciertos públicos y privados para aprovechar la totalidad de los recursos sanitarios disponibles en el país. Este posicionamiento abrió un encendido debate político y social entre los defensores de la medicina liberal y los partidarios de la medicina social, lo que no benefició el avance hacia la reforma consensuada. Es más, los dos frentes mantuvieron sus posiciones durante los ańos de transición y, lejos de buscar el acuerdo, convirtieron la controversia en materia de salud en uno de los principales enfrentamientos políticos parlamentarios.

26 «Proyecto para centralizar los ingresos y gastos de la Seguridad Social», El País, 31-5-1978.

27 «El difícil e inalcanzable camino de la reforma sanitaria», El País, 15-9-1978.

28 Elola (2001): 53. 
A medida que pasaba el tiempo, el INP resultaba más cuestionado por la opinión pública, los colegios profesionales, sindicatos y parte de los políticos. Sin embargo, el ministro de Sanidad continuaba apoyando públicamente su gestión y lo calificaba de «organismo ideal para actuar como soporte de nuevas andaduras, debido a su capacidad para adaptarse a las nuevas circunstancias sociales ${ }^{29}$. Pero el mantenimiento del INP se había convertido en un escollo para avanzar, por lo que estaba condenado a desaparecer. Los cambios no llegaron hasta la aprobación de la Constitución en 1978. Sin duda, había demasiados frentes abiertos en el período y era necesario establecer prioridades. La Constitución dedica a la salud el art. 43, redactado como sigue ${ }^{30}:$ «1. Se reconoce el derecho a la protección de la salud; 2 . Compete a los poderes públicos organizar y tutelar la salud pública a través de medidas preventivas y de las prestaciones y servicios necesarios. La ley establecerá los derechos y deberes de todos al respecto; 3 . Los poderes públicos fomentarán la educación sanitaria». Este artículo sienta las bases legislativas de un enfoque más moderno y europeísta de la sanidad en España, pero a la vez deja un amplio campo a las reformas sanitarias. Por otro lado, la Constitución contiene también reglas básicas sobre la distribución de las competencias sanitarias entre el Estado y las comunidades autónomas. Según el art. 149.1.16.a, corresponde al Estado «la Sanidad exterior. Bases y coordinación general de la sanidad. Legislación de productos farmacéuticos». Este artículo abría la posibilidad de caminar hacia la descentralización sanitaria.

Una vez conseguido el acuerdo constitucional, en el otońo de 1978, se aprobó el Real Decreto Ley 36/1978 ${ }^{31}$, por el que se procedió a la reorganización de todo el sistema de Seguridad Social con el fin de simplificar y racionalizar su labor. Dentro de este contexto, desapareció el INP, se extinguieron el Servicio de Mutualismo Laboral, las mutualidades laborales y demás instituciones de estructura mutualista, y se crearon tres grandes entidades gestoras de la Seguridad Social, sujetas a principios de solidaridad financiera y unidad de caja ${ }^{32}$ : El Instituto Nacional de la Seguridad Social (INSS), gestor de las prestaciones económicas, donde quedaron integradas las mutualidades y

29 «La seguridad social debe reformarse en profundidad», $A B C, 28-2-1978$.

30 Muñoz et al. (1997): 223. Sobre la Constitución de 1978 y el modelo económico y social de Estado, véase Vallejo (2003).

31 BOE 18/11/1978, núm. 276, pp. 26.245-26.249.

32 El Mutualismo Laboral había nacido con la aprobación de la Orden del Ministerio de Trabajo de 24 de octubre de 1946 con el objetivo de complementar los seguros sociales existentes, dado que la cuantía de sus prestaciones se había mantenido muy baja. Más detalle sobre este tema tan complejo en Pons y Vilar (2014): 145 y ss. 
demás entidades gestoras de estructura mutualista ${ }^{33}$; el Instituto Nacional de Salud (INSALUD), para la administración y gestión de los servicios sanitarios; y el Instituto Nacional de Servicios Sociales (INSERSO), responsable de los servicios complementarios de las prestaciones del Sistema de la Seguridad Social (como los servicios a ancianos y personas inválidas). En paralelo, como organismos autónomos del Estado, se crearon la Administración Institucional de la Sanidad Nacional, para la administración de los servicios de prevención y asistencia que le fuesen encomendados, y el Instituto Nacional de Asistencia Social, para ejecutar los servicios de asistencia social del Estado, complementarios a los del Sistema de Seguridad Social. Dentro de este organismo quedaron integrados los establecimientos de asistencia pública, dependientes de la Dirección General de Servicios Sociales. De manera simultánea, quedaron transferidas a la Administración institucional del Estado y dejaron de tener la condición de Organismos de la Seguridad Social: el Servicio de Empleo y Acción Formativa, Promoción Profesional Obrera, el Servicio de Universidades Laborales y el Servicio Social de Seguridad e Higiene en el Trabajo. Junto con estas medidas, se crearon también el Instituto Nacional de Empleo para gestionar las prestaciones del desempleo y la Tesorería General de la Seguridad Social, responsable de administrar los recursos del sistema. En conjunto, con estos cambios se pusieron las bases para unificar todas las competencias en materia de salud en torno a una única entidad, el INSALUD ${ }^{34}$.

En la introducción del Real Decreto Ley 36/1978 se destacan tres principales problemas - heredados del franquismo- que justifican los cambios en la gestión de la sanidad española. En primer lugar, era necesario simplificar el número de entidades gestoras, racionalizar sus funciones y descentralizar sus tareas administrativas. Además, el texto «faculta al Gobierno para regular la participación en ellas de sindicatos, organizaciones empresariales y Administración, dando así cumplida respuesta a una de las demandas sociales de mayor arraigo». En segundo lugar, la reforma permite reintegrar al Estado funciones que había asumido la Seguridad Social y que no son propias de la misma, «tales como las referidas a empleo, educación y servicios sociales, más propias de un concepto de servicio público que no del delimitado acotamiento de prestaciones de la Seguridad Social». En tercer lugar, la pretensión de

33 Recordemos que en los años setenta se aprobaron diversas mutualidades de funcionarios que generaron efectos positivos en el negocio del seguro privado de salud. La entrada en vigor de la Ley 33/1984, de 2 de agosto, de Ordenación de Seguro Privado determinó la inclusión de las mutualidades y montepíos de previsión social en el sector de los seguros; véase Pons y Vilar (2014): 400.

34 Sevilla (2006): 14. 
simplificación y racionalización se "compatibiliza con el principio de caja única en toda la Seguridad Social». En conjunto, la reforma posibilita una clara delimitación de tres áreas diferentes con objetivos específicos: la salud, la seguridad social y los servicios de asistencia social.

En septiembre de 1978 un artículo en la Tribuna Libre de El País ponía en evidencia la insostenible situación de la asistencia sanitaria en España basándose en cuatro deficiencias ${ }^{35}$ : 1) ausencia de una medicina básica urbana y rural con ambulatorios desbordados y carentes de medios adecuados; 2) escasez e irracionalidad en la distribución de hospitales; falta de medidas preventivas mínimas y de educación sanitaria; 3) abusivo consumo de fármacos; 4) nefasta gestión económica del INP, que carecía de la menor participación democrática de los trabajadores sanitarios y los asegurados, y presenta graves irregularidades con los fondos «que en ocasiones se han dedicado a subvencionar sectores poco rentables del INI, e incluso a reservas disponibles para la banca privada». La parálisis legislativa no hacía más que agravar los problemas.

\section{EL DEBATE SOBRE EL MODELO SANITARIO EN LOS PRIMEROS PASOS DE LA DEMOCRACIA (1979-1981)}

La aprobación de la Constitución y la creación de las entidades gestoras de la Seguridad Social no desactivaron el conflicto en torno a la renovación sanitaria, principal objetivo de los responsables de la cartera de este ministerio en las siguientes legislaturas. Unos meses más tarde, tras las elecciones de marzo de 1979, Sánchez León fue sustituido en la cartera de Sanidad sin haber conseguido aprobar una ley de sanidad y con la limitada satisfacción de haber sustituido al obsoleto y cuestionado INP por un nuevo sistema de gestión de la Seguridad Social. El reparto de los escaños en el Congreso entre las principales fuerzas políticas (UCD, 168; PSOE, 121; PCE, 23 y AP/PP, 9) apenas había variado respecto a las elecciones de 1977, pero la oposición política del PSOE a las políticas gubernamentales se recrudeció en un marco de crecientes expectativas electorales ${ }^{36}$.

Al igual que su predecesor, Juan Rovira Tarazona (abril 1979-septiembre 1980), ministro de Sanidad y Seguridad Social en la primera legislatura constitucional, se propuso como principal objetivo de su mandato la elaboración de

35 «La insostenible situación de la asistencia sanitaria», El País, 8-9-1978, Hemeroteca Archivo Linz, R-59748.

36 Datos recogidos de Linz et al. (2005), cuadro 14.26, p. 1.114. 
dos nuevas leyes sobre seguridad social y sanidad ${ }^{37}$. En una de sus primeras conferencias de prensa el ministro Rovira planteaba tres medidas urgentes a conseguir: la puesta en funcionamiento de la Tesorería de la Seguridad Social, «con el fin de clarificar los temas financieros pendientes y gestionar mejor los recursos»; promocionar los nuevos institutos recientemente creados; y presentar al Congreso lo antes posible los nuevos textos legislativos ${ }^{38}$. Un mes más tarde, en junio de 1979, se hacen públicos dos textos, uno del Gobierno y otro del principal partido de la oposición, el PSOE, que ponen sobre la mesa una propuesta de corte muy diferente ${ }^{39}$. El acuerdo resultó imposible. Por aquel entonces España necesitaba una reforma sanitaria de Estado, pero el tema de la sanidad se había convertido en un pulso entre las dos principales fuerzas políticas.

A finales de diciembre de 1979, una comisión de expertos elegida en el Congreso de los Diputados presentó un índice básico de temas para comenzar a trabajar en el proyecto sanitario. La comisión, integrada por catorce miembros, entre los que figuraban el secretario de Estado para la Sanidad; el director general de Salud Pública; los presidentes de los Consejos Generales de los Colegios Médicos, Farmacéuticos, Veterinarios, Técnico-Sanitarios y Odontólogos; los decanos de las facultades de Medicina de Madrid y Barcelona y varios catedráticos de Derecho Administrativo, pasó el testigo a un grupo de trabajo integrado por representantes de todos los grupos parlamentarios para confeccionar un borrador de reforma sanitaria a partir del índice propuesto ${ }^{40}$. Dos meses más tarde, se presentó un primer borrador que tuvo mala acogida ante la Comisión de Sanidad del Congreso de los Diputados, presidida por el antiguo ministro Sánchez León - lo que ponía en evidencia la línea continuista de la UCD—, sobre todo por el grupo parlamentario socialista ${ }^{41}$.

En febrero de 1980 se dieron por terminados los debates previos en la Comisión sobre las líneas generales de la reforma sanitaria, sin haber alcanzado el consenso político. ¿Dónde estaba el principal escollo? De nuevo, en el

37 Abogado del Estado que ocupó diversos cargos en el Ministerio de Hacienda durante la dictadura. Más tarde, en 1976, fue nombrado subsecretario de la Seguridad Social y luego ministro de Sanidad. Tras su cese en 1980, pasó a ser delegado del Gobierno en Cataluña. Información obtenida del Archivo del Congreso de los Diputados.

"Rovira con los delegados territoriales de sanidad», $A B C, 30-5-1979$.

39 "Reforma sanitaria», $A B C, 30-6-1979$.

40 "No hay consenso en la financiación ni sobre el servicio nacional de salud», $A B C$, 1-12-1979.

41 «La comisión ejecutiva del PSOE estudió la reforma sanitaria», Diario 16, 30-1-1980, Hemeroteca Archivo Linz, R-20061, y «El PSOE contra el documento de la reforma sanitaria», $A B C, 13-2-1980$. 
diferente modelo sanitario que pretendían la UCD y el PSOE. Para la UCD, la sanidad no debía ser más que una tarea del Estado ejercitada no a través de un Servicio Nacional de Salud, «que no es más que la estatalización de la gestión sanitaria», sino de un servicio público en el que puedan concurrir todos los agentes sanitarios incluidos los de la iniciativa privada. Mientras tanto, los socialistas partían de tesis estatalizadoras y proponían un profundo proceso de reforma sanitaria que supusiera una auténtica renovación del modelo existente $^{42}$. Ambos partidos cruzaron acusaciones de plagio, mala fe e incitación al disenso en la prensa del país. El proceso se enquistó cada vez más para un Ejecutivo que no contaba con la mayoría absoluta en las Cortes y un PSOE que veía cada vez más cerca su llegada al Gobierno ${ }^{43}$.

En abril de 1980, la federación de sanidad de UGT hizo pública una propuesta de ley de sanidad con cuatro garantías básicas no negociables: la protección de la salud de todos los españoles y no solo de los que pagasen cuotas a la Seguridad Social; que las obligaciones concretas del sector sanitario recayesen en los municipios; la necesaria participación de los usuarios en los órganos de gestión; y, por último, una financiación presupuestaria para el sector ${ }^{44}$. La propuesta recibió una buena acogida en el ámbito socialista, pero chocó con algunos de los planteamientos básicos expuestos por el Gobierno. Un mes más tarde, en mayo de 1980, el Congreso retomó el debate sobre la reforma sanitaria a partir del texto elaborado por la Comisión de Sanidad y Seguridad Social de la Cámara. Su presidente, Sánchez León, insistía en la idea de que «la sanidad no debe ser más que una tarea del Estado, ejercitada no a través de un Servicio Nacional de Salud, que no es más que la estatalización de la gestión sanitaria, sino de un servicio público de salud en el que puedan concurrir todos los agentes sanitarios, incluidos los de la iniciativa privada ${ }^{45}$. El PSOE presentó una enmienda a la totalidad del texto elaborado por la UCD al que calificó de "chapuza continuista». La enmienda se rechazó y el debate otra vez se enquistó.

La propuesta de bases para la reforma sanitaria presentada por la UCD siguió adelante, apoyada por el partido liderado por Fraga Iribarne, Coalición Democrática. El siguiente paso sería la elaboración de un proyecto de ley y su posterior remisión al Parlamento. Pero, otra vez, el proyecto se vio frenado por la sombra de las irregularidades y las confrontaciones políticas. De un lado, la

\footnotetext{
42 «El Ministro de Sanidad defendió la reforma sanitaria», $A B C, 8-3-1980$ y «La Comisión de Sanidad y Seguridad Social», $A B C, 20-3-1980$.

43 «El Ministerio de Sanidad acusa de plagio al PSOE», $A B C$, 21-2-1980.

44 «UGT elabora una ley de sanidad», $A B C, 3-4-1980$.

45 «El Congreso comienza a estudiar hoy la reforma sanitaria», $A B C, 6-5-1980$.
} 
oposición liderada por el PSOE presionaba por una reforma sanitaria «de Estado». De otro, salía a la luz el escándalo por la filtración de unas auditorías e informes internos que ponían de relieve presuntas irregularidades en numerosos hospitales públicos ${ }^{46}$. Dentro de este contexto, en septiembre de 1980, se produjo un relevo ministerial. Alberto Oliart Saussol llegó al Ministerio de Sanidad con el firme propósito de impulsar una comisión parlamentaria para investigar a fondo y clarificar las cuentas del INSALUD ${ }^{47}$. Para poner orden en la Seguridad Social, incorporó a su equipo al economista José Barea. El proceso creó fuertes tensiones en los «cuadros históricos» del citado organismo y un cruce de declaraciones en la prensa, aprovechado por diferentes sectores políticos y profesionales ${ }^{48}$.

Oliart trató de aportar soluciones al peligroso agujero financiero de las cuentas de la Seguridad Social, pero la necesidad de medidas de ajuste para afrontar la gravedad de la crisis vigente dejó a la sanidad en segundo plano. En palabras del ministro, «las únicas expectativas consisten en aumentar la presión fiscal o elevar las cuotas de la Seguridad Social. Ambas soluciones nos llevarían a un callejón sin salida desde un punto de vista económico, por lo que mi propuesta es un mayor control del gasto y mayor eficacia en la administración de los recursos» ${ }^{49}$. Para dar respuesta a estos problemas, el ministro solicitó la vía del consenso a las demás fuerzas políticas pero, dados los precedentes, había poco espacio para la esperanza. En paralelo, el Gobierno contaba cada vez con menos apoyo parlamentario y mediático, lo que derivó en la dimisión de Suárez en enero de 1981.

El nuevo Gobierno de Leopoldo Calvo-Sotelo nombró como ministro de Sanidad y Consumo a Jesús Sancho Rof, quien tenía ante sí una tarea complicada, en un marco político de crispación general golpeado por la crisis del intento de golpe de Estado del 23-F y una crisis económica pendiente de

46 «Rovira informa del fraude a la Seguridad Social», $A B C$, 19-6-1980.

47 Abogado del Estado, ocupó cargos en Renfe desde los años sesenta. En 1973 dejó la Administración para ser consejero del Banco Hispano Americano y del Banco Urquijo, entre otras empresas privadas. En 1977 fue nombrado ministro de Industria y Energía; en 1981, ministro de Sanidad y, tras el golpe de Estado, Calvo-Sotelo lo nombró ministro de Defensa. Información obtenida del Archivo del Congreso de los Diputados.

48 «UCD no ha desarrollado la reforma sanitaria aprobada en 1980», El País, 02-10-1982.

49 «Oliart propugnó el control del gasto de la seguridad social», $A B C, 23-1-1981$ y "Alberto Oliart expone la situación de la asistencia sanitaria de nuestro país", $A B C$, 25-1-1981. 
$\operatorname{cerrar}^{50}$. En paralelo, las tensiones internas en el seno del Ministerio crecían, a la sombra de la corrupción vigente en el ámbito sanitario y la pesada herencia de cuarenta años de errores acumulados en el sistema. El debate parecía en punto muerto. La prensa de los primeros ańos ochenta repetía los mismos enfrentamientos y conflictos en torno a la reforma sanitaria: las reivindicaciones del Sindicato de Médicos Libres, las amenazas de huelga de los colegios profesionales médicos y farmacéuticos y las posturas encontradas de Gobierno y oposición en un marco de deterioro progresivo de la sanidad pública. Ahora bien, en esta etapa la brecha entre los modelos sanitarios propuestos por los diferentes partidos políticos era prácticamente insalvable.

El ministro Sancho Rof aclaraba que aunque la Constitución dice que «el sistema de Seguridad Social es un sistema público, único para todos los españoles», esto no implicaba que el Estado tuviera que ejercer como principal actor del sistema sanitario ${ }^{51}$. El ministro continuaba defendiendo un modelo basado en conciertos con la sanidad privada; pues lo contrario sería un despilfarro de recursos. Dentro de este marco, algunos miembros de la UCD llegaron a afirmar que "la Seguridad Social no sirve y que hay que buscar un nuevo gestor, que no es otro que la iniciativa privada ${ }^{52}$. Mientras tanto, el PSOE (representado por el diputado Vicente Martín) acusaba al Gobierno de contribuir a la degradación de la sanidad pública a través de la congelación de la oferta material y humana y la paralización de inversiones para facilitar su privatización ${ }^{53}$. El grupo socialista proponía reformas profundas dentro de un modelo de sanidad pública en un marco de denuncia del «despilfarro de recursos, incumplimiento de horarios y dedicación, incompatibilidades, creación de feudos privados en centros públicos, conciertos descontrolados que se traducen en la utilización de camas ajenas, cuando se dispone de propias no utilizadas $[\ldots])^{54}$.

50 Sancho Rof fue director general de RTVE en los últimos ańos de la dictadura y ocupó varias carteras ministeriales en los Gobiernos de la UCD presididos por Suárez y por Calvo-Sotelo. Información obtenida del Archivo del Congreso de los Diputados.

51 Congreso de los Diputados, Sesiones Informativas de Comisiones, Acta taquigráfica de la sesión celebrada por la Comisión de Sanidad y Seguridad Social, jueves 23 de abril de 1981, núm. 29, p. 3.

52 "En el fondo de un conflicto hay un asalto a la Seguridad Social», $A B C, 8-4-1981$.

53 Congreso de los Diputados, Sesiones Informativas de Comisiones, Acta taquigráfica de la sesión celebrada por la Comisión de Sanidad y Seguridad Social, jueves 23 de abril de 1981, núm. 29, pp. 24-25.

54 «En el fondo de un conflicto hay un asalto a la Seguridad Social», $A B C, 8-4-1981$. 
En un marco de debilitamientos y desintegración de la UCD y de fortalecimiento y creciente popularidad del proyecto socialista, la reforma sanitaria permaneció en segundo plano hasta caer en el olvido, tras el estallido del «síndrome tóxico» del aceite de colza, que supuso un mayor desprestigio para la sanidad española. Este nuevo escándalo puso de manifiesto «la artificiosidad administrativa de un ministerio sometido a sucesivas reestructuraciones, a un cambio constante de competencias y de equipos rectores, así como las luchas intestinas interministeriales e intercorporativistas en función de la interrelación de competencias ${ }^{55}$.

La etapa de la transición se cerraba con un nuevo ministro de Sanidad. A finales de 1981, Manuel Núnez Pérez volvía a poner sobre la mesa un paquete de medidas para mejorar y racionalizar la Seguridad Social, «las mismas que se han venido prometiendo desde hace años, sin que se llegaran a cumplir $»^{56}$. Las críticas a la gestión ministerial se repetían en la prensa en duros artículos que señalaban que "parece una burla el que en 1982 se nos vuelva a prometer lo ya prometido en 1978 y 1980, sin haber abordado el único objetivo realizable y barato: un servicio integral y justo de salud ${ }^{57}$. El hecho de haber contabilizado cuatro ministros de sanidad en cuatro años ponía de manifiesto las dificultades de la reforma sanitaria en España, el juego de intereses externo e interno al Ministerio y el fracaso de la transición en esta parcela. Núñez impulsó la Ley de incompatibilidades del personal al servicio de la Seguridad Social, un proyecto que encendió todavía más el debate entre el Gobierno y la oposición. En las vísperas de una nueva cita electoral, en octubre de 1982, el ministro de Sanidad anunciaba la elaboración de una nueva normativa para regular y racionalizar el sistema de conciertos entre centros privados y la Seguridad Social para la asistencia de enfermos. Unos días más tarde la UCD perdía las elecciones. El balance en cifras de los primeros Gobiernos democráticos en el ámbito de la sanidad resulta bastante pobre ${ }^{58}$. El porcentaje de población cubierta apenas había variado entre $1975(81 \%)$ y 1980 (83 \%), por lo que la cobertura universal permanecía como un objetivo a conseguir. La financiación del gasto sanitario público procedía fundamentalmente de las cotizaciones de empresarios y trabajadores, mientras que el Estado aportaba apenas el $25 \%$ en 1980. El gasto total en salud como porcentaje del PIB situaba a España en el vagón de cola europeo (1980: 5,3 \%), por

\footnotetext{
55 «UCD no ha desarrollado la reforma sanitaria aprobada en 1980», El País, 2-10-1982.

56 «Seguridad Social y el desconcierto», El Pais, 11-4-1982.

57 «Seguridad Social y el desconcierto», El Pais, 11-4-1982.

58 Estas cifras han sido obtenidas de Pons y Vilar (2014), cuadros 4.1, 4.2, 4.3 y 4.7.
} 
detrás de otros países como Grecia y Portugal. En conjunto, en 1982 el consumo final de sanidad en España era provisto en un 79,2 \% por la sanidad pública y el resto por la sanidad privada. Detrás de estos porcentajes había muchos intereses contrapuestos que se trataron de integrar para conseguir aprobar la ley de sanidad.

\section{LA APROBACIÓN DE LA ANSIADA LEY DE SANIDAD (1986): DEBATE Y CONSECUENCIAS}

Las elecciones celebradas en 1982 dejaron un reparto de escaños en el Congreso muy diferente a las elecciones anteriores. De un lado, el PSOE obtuvo la mayoría absoluta con 202 diputados y, de otro, AP/PP se erigió como principal partido de la oposición (107); mientras que UCD (11) y el PCE (4) perdían peso en la Cámara de Diputados ${ }^{59}$. Cuando el PSOE llegó al poder en octubre de 1982, la reforma de la sanidad española constituía un trabajo pendiente. Desde el primer momento quedó claro que la estrategia del PSOE en este ámbito iba a ser muy diferente desde los bancos azules del Gobierno. Su objetivo principal se centró en lograr aprobar la Ley de Sanidad, algo que no habían conseguido los gobiernos anteriores, aunque esto implicase la renuncia a algunos elementos básicos de su proyecto inicial.

Recordemos que el PSOE había propugnado desde la oposición en los años de gobierno de la UCD un sistema sanitario tipo Servicio Nacional de Salud, del estilo a los implantados en otros países europeos, sostenido sobre tres principios básicos: de cobertura universal por la condición de ciudadano, financiado mayoritariamente vía impuestos y de gestión pública ${ }^{60}$. La puesta en marcha de este proyecto, una vez alcanzado el Gobierno, debía hacer frente a dos problemas principales: la crisis económica, que limitaba los recursos, y la oposición de los partidos políticos y los sectores sanitarios más conservadores. Tampoco podemos olvidar las eternas herencias sanitarias en forma de opacidad financiera y gestora. El recién iniciado proceso de traspaso de competencias de sanidad en 1981 a las comunidades autónomas añadía algunas dificultades. A pesar de ello, el ejecutivo preveía aprobar la reforma sanitaria de manera rápida, aunque el proceso se prolongó más de lo esperado. La aprobación de la Ley General de Sanidad hubo de esperar hasta casi el fin de la legislatura en 1986 y solo se consiguió con un proyecto descafeinado que

59 Datos recogidos de Linz et al. (2005), cuadro 14.26, p. 1114.

60 Sevilla (2006): 14. Sobre las novedades de la propuesta de modelo sanitario del PSOE, véase Barbado y Powell (2011). 
trataba de integrar en mayor o menor medida los intereses de todas las partes (políticos, empresarios, sociedad y profesionales).

\section{EL DEBATE SOBRE LA LEY GENERAL DE SANIDAD}

En febrero de 1983, en una de sus primeras ruedas de prensa como ministro de Sanidad y Consumo, Ernest Lluch declaraba a los periodistas ${ }^{61}$ : «Nos hemos encontrado en el Instituto Nacional de la Salud con una situación de poca claridad financiera» ${ }^{62}$. El ministro continuaba señalando que «aparecen con frecuencia deudas de las que no se había recibido información y este organismo se encuentra en estos momentos con una gran necesidad de clarificar totalmente el estado de las cuentas». En junio de 1983 Lluch presentó su proyecto de reforma sanitaria, inspirado en un programa de universalización y racionalización de la sanidad, en el marco de unas jornadas internacionales convocadas exclusivamente para analizar los puntos esenciales que debería incluir la nueva configuración sanitaria de un país que pretendía estar en esta materia a la altura de sus vecinos europeos.

Como siguiente paso, se nombró una comisión encargada de redactar el borrador inicial, formada por el subsecretario de Sanidad, Pedro Sabando; el catedrático de Derecho Administrativo Pedro Muñoz; los consejeros de Sanidad de los Gobiernos de Andalucía, País Vasco y Madrid; dos representantes de la Federación Española de Municipios; tres directores generales del Ministerio de Sanidad; el secretario general técnico, el director del gabinete del ministro y el asesor ejecutivo, Pedro Pablo Mansilla ${ }^{63}$. Diez meses más tarde, Lluch hacía público el primer borrador del anteproyecto de ley básica de Sanidad, donde se ponía de manifiesto que de las «tres patas básicas de la sanidad, la medicina hospitalaria se encuentra a buen nivel, en la medicina primaria existe retraso y muchas disfunciones y en la medicina preventiva, un gran retraso ${ }^{64}$. Entre las

61 En 1977 fue elegido diputado en las elecciones a Cortes Constituyentes, como cabeza de lista de Socialistes de Catalunya; reelegido en 1979 y en 1982. En mayo de 1986 se retiró del primer plano político y volvió a su cátedra de Economía en la Universidad de Barcelona. Fue asesinado por ETA en el año 2000. Su último cargo oficial fue como rector de la Universidad Internacional Menéndez Pelayo de Santander entre 1989 y 1995. Datos biográficos obtenidos de la Fundación Ernest Lluch.

62 «Las finanzas del Insalud son poco claras, según Ernest Lluch», El País, 4-2-1983.

63 «Ocho meses de consultas y negociación», El País, 25-10-1984.

64 «Ernest Lluch hará público hoy el contenido del anteproyecto de ley básica de Sanidad que se enviará en enero a las Cortes», El País, 27-12-1983. 
principales ideas recopiladas en el texto podemos destacar «la extensión de la cobertura sanitaria a toda la población, que se realizará de forma progresiva, y la creación de un Servicio Nacional de Salud que se adapta a la estructura estatal autonómica ${ }^{65}$.

Pero el modelo sanitario propuesto inicialmente por el PSOE desde los escańos de la oposición en las anteriores legislaturas había quedado muy desfigurado con el fin de integrar los intereses de todas las partes y lograr aprobar la ley con el mayor consenso posible. Así se ponía de manifiesto en el discurso del ministro Ernest Lluch durante la discusión del proyecto de ley de sanidad en el Congreso en $1985^{66}$. Su proyecto perseguía «mantener una relación estable entre la sanidad pública y privada» dentro de las directrices del sector público (demanda de sector privado). Y añadía que «la mayor parte del sector privado hospitalario de este país se mueve en relación con el sector público, es decir, que sin tener flujos públicos no podría subsistir. Solo el $17 \%$ de los servicios privados de la sanidad no están concertados con el sector público». Su proyecto proponía la universalización de las prestaciones (demanda social) y la creación de más empleo en el ámbito sanitario, libre ejercicio de la profesión médica y mejora de las condiciones laborales (demanda de los sindicatos y colectivos profesionales). Por último, el ministro señalaba en su discurso que en España «no se podía establecer un Servicio Nacional de Salud», puesto que estamos ante un sistema con «autonomías políticas de los servicios», aunque el Estado debe proporcionar unas garantías mínimas para todos los españoles (demanda de las comunidades autónomas). Además, el proyecto proponía el «mantenimiento de un mecanismo mixto de financiación donde las cotizaciones sociales se seguían contemplando como una fuente de financiación", aunque con el deseo de ir aumentando de manera progresiva la aportación del Estado. La exposición del proyecto de ley se encontró con la oposición de AP/PP y el PCE, aunque desde enfoques y estrategias muy diferentes. El único consenso de todos los grupos parecía estar en la necesidad de una reforma sanitaria y en los problemas graves (financieros, de gestión y atención) de la sanidad española.

De un lado, el diputado José María Romay Beccaria, en representación de AP, puso énfasis en criticar «la mala asistencia sanitaria pública» y en la necesidad de dar «libertad a los usuarios para elegir los servicios que desean»; es decir, defendía la plena libertad de elección de médicos y centros hospitalarios (públicos o privados) y la firma de conciertos para conseguir este

65 «La ley general de Sanidad ampliara la cobertura médica a toda la población y unificará las redes asistenciales», El País, 28-12-1983.

66 Diario de Sesiones del Congreso de los Diputados, II Legislatura, 1985, núm. 215, sesión plenaria núm. 215, pp. 9852-9855. 
objetivo $^{67}$. Carlos Ruiz Soto, también diputado popular, complementaba este discurso afirmando que "el Servicio Nacional de Salud es una antigualla que está en solfa» y proponía "hacer complementarias las dos medicinas, las dos sanidades, la pública y la privada, pero no complementarias una de la otra, porque además en nuestra Constitución está también al libertad de empresa». De otro lado, el diputado del PCE y portavoz del Grupo Mixto, Fernando Pérez Royo, defendió su enmienda a la totalidad del proyecto basándose en su defensa de un servicio público sanitario con las características que históricamente han definido al Servicio Nacional de Salud. Y continuó su argumento: «En contra de esta alternativa sanitaria solo ha estado la cúpula de la organización médica colegial, los sectores empresariales de la medicina privada, una parte de la patronal farmacéutica y los sectores financieros interesados en la puesta en marcha de seguros complementarios de enfermedad». Al final de su discurso, criticó los pasos atrás dados por el PSOE en su proyecto sanitario y su abandono del proyecto de Servicio Nacional de Salud (SNS). En la réplica, Vicente Martín, diputado del PSOE, sostuvo que un SNS ya no era jurídicamente viable, pero defendió la idea de que «el Servicio Nacional de la Salud de las Comunidades Autónomas, con carácter progresista, integrador, publico, generalizado...» ya está incluido en el proyecto de ley presentado.

A continuación, se abrió un período de recopilación de críticas y sugerencias por parte de los agentes institucionales, sociales y profesionales. La entrada en vigor de la nueva ley se demoró, no solo por la necesidad de encontrar vías de acuerdo entre los diferentes agentes políticos, profesionales y sociales, sino también por las discrepancias internas en el propio gobierno. De un lado, la resistencia numantina de los sectores pro-seguros sociales a la aprobación de la Ley General de Sanidad, encabezados por Alianza Popular y los colegios médicos ${ }^{68}$. De otro, la extensión de la protección a todos los ciudadanos (en 1985 quedaban fuera de la cobertura alrededor de dos millones de personas), la concepción de la salud como un servicio integral (hasta el momento limitado a atender la enfermedad) y la ampliación de la cobertura sanitaria a nuevos campos (psiquiatría, venta de productos terapéuticos y orientación familiar, principalmente) se traducía en mayor gasto, lo que generó reticencias con el Ministerio de Hacienda presidido por Miguel Boyer ${ }^{69}$. La necesidad de encontrar nuevas vías de financiación para esta partida dividió al Ejecutivo. El

67 Para este párrafo, véase Diario de Sesiones del Congreso de los Diputados, II Legislatura, 1985, sesión plenaria núm. 215, pp. 9856-9901.

68 Elola (2001): 130. Sobre el proceso político que condujo a la aprobación de la Ley General de Sanidad de 1986, véase Giménez (2011).

69 «Lluch y Boyer aproximan posiciones sobre la reforma sanitaria», El Pais, 6-2-1985. 
consenso interno fue facilitado por el XXX Congreso del PSOE, que consideró urgente la reforma y manifestó el propósito de elevar en los próximos años los gastos sociales por encima del crecimiento de la actividad económica. Para este fin, el Estado debería aumentar de manera paulatina la aportación a la Seguridad Social durante unos ańos, con dos fines principales: pagar la extensión del servicio a todos los ciudadanos y conseguir la integración en la red pública de asistencia de las diversas administraciones sanitarias, hasta ahora dependientes de las diputaciones y ayuntamientos, incluyendo toda la red de beneficencia y casi todas las instalaciones psiquiátricas existentes.

Tras un proceso tormentoso de más de tres años, donde se sucedieron complicadas negociaciones con diversos sectores políticos, fuerzas sociales y profesionales, se aprobó la Ley General de Sanidad en 1986, que abordó la difícil tarea de sentar al mismo tiempo las bases de dos procesos complejos: la modernización de la sanidad española y la descentralización de su gestión. Sin embargo, el texto no satisfizo a casi nadie. La derecha tachaba la ley de dirigista y le achacaba fundamentalmente no instaurar la libre elección de médico y de sistema sanitario ${ }^{70}$. Los sectores progresistas criticaban que la ley no instaurase un servicio nacional de salud, al estilo del británico, y que no garantizase la gratuidad total de la prestación sanitaria y, en consecuencia, su universalidad. En particular, la prensa calificaba el texto de «totalmente descomprometido» y virtual, pues se condicionaba la práctica de la ley a circunstancias de subjetiva interpretación como «el proceso evolutivo de los costes» $\mathrm{O}$ «las disponibilidades presupuestarias» $»^{71}$.

En realidad, la Ley General de Sanidad (LGS) 14/1986, de 25 de abril, aprobada finalmente con los votos a favor de PNV, PSOE, PCE y CiU, supuso el desarrollo legislativo estatal del derecho a protección de la salud establecido en las bases de la Constitución. Sin embargo, este texto contenía más un conjunto de principios y objetivos de largo alcance que un plan de ejecución inmediato de reforma sanitaria ${ }^{72}$.

\section{EL MODELO ESTABLECIDO POR LA LEY GENERAL DE SANIDAD}

La LGS establecía que la asistencia sanitaria pública se extendería a toda la población española en condiciones de igualdad (art. 3.2) y que la actuación de

\footnotetext{
70 «El cambio apenas ha tocado la estructura sanitaria», El País, 10-6-1986.

71 «La ley general de Sanidad, una reforma a la inversa», El País, 20-9-1985.

72 Muñoz et al. (1997): 224. La ley completó también el desarrollo de la reforma de la atención primaria iniciada con el Real Decreto 137/1984, BOE 01/02/1984, núm. 27, pp. 2627-2629.
} 
las Administraciones públicas sanitarias estaría orientada a promover la salud en general, fomentar la educación sanitaria de la población, garantizar acciones de prevención y curar enfermedades, asegurar la asistencia sanitaria en caso de pérdida de salud y promover acciones para la rehabilitación y reinserción social del paciente (art. 6). La cobertura sanitaria pública en España pasó del $81 \%$ de la población en 1975, al $90 \%$ en 1985 y al $99 \%$ en 1990, tras la incorporación de la población atendida hasta el momento por los servicios de beneficencia ${ }^{73}$. El denominado Decreto de Universalización ${ }^{74}$ puso fin en España a la caridad sanitaria de la antigua beneficencia y dio acogida en el SNS a las personas sin recursos económicos. En general, la ampliación de la cobertura benefició al menos a tres sectores importantes: los parados, que tenían garantizada indefinidamente la asistencia sanitaria de su familia; los jóvenes sin empleo, atendidos hasta los 26 años; y los trabajadores autónomos, que quedaron también incorporados en el régimen general. Además, desde 1984 las mutualidades de funcionarios (MUFACE, MUGEJU, ISFAS) gozaron de la posibilidad de elegir cada año el aseguramiento (SNS o compañías de seguro privado), mientras que antes se adscribían de manera obligatoria a una compañía privada. Ahora bien, la universalización de la sanidad española exigía un incremento de gasto sanitario público y una transformación del modelo de financiación.

En segundo lugar, la LGS configuró una estructura mixta de financiación para el Servicio Nacional de Salud procedente de: cotizaciones sociales, transferencias del Estado, tasas por la prestación de determinados servicios y aportaciones de las comunidades autónomas y corporaciones locales (art. 79). Establecía además que los «presupuestos del Estado, Comunidades Autónomas, Corporaciones Locales y Seguridad Social consignarán las partidas precisas para atender las necesidades sanitarias de todos los organismos e instituciones dependientes de las Administraciones Públicas y para el desarrollo de las competencias» (art. 78). No obstante, el modelo diseñado de SNS apostaba por el incremento progresivo de la financiación del sistema en base a impuestos generales en lugar de cotizaciones a la Seguridad Social, como venía ocurriendo hasta el momento. A partir de la modificación del sistema de financiación de la asistencia sanitaria, introducida en la Ley de Presupuestos Generales del Estado 37/1988 para 1989, la mayor parte de la financiación de la sanidad se produce a través de los ingresos del Estado ${ }^{75}$. Dentro de este proceso, el peso de las cotizaciones sociales y de la financiación estatal cambió de manera progresiva entre 1980 y 1999, año en el que se aprobaron los primeros

73 Información obtenida de Pons y Vilar (2014): cuadro 4.1.

74 Real Decreto 1088/89, BOE 09/09/1989, núm. 216, pp. 28657-28658.

75 Temes y Gil (1996): 12. 
presupuestos que incluían una financiación completa de la sanidad a partir de impuestos. De este modo, el porcentaje de financiación del gasto público por parte del Estado creció desde el 24,8 \% en 1980 al 72,8 \% en $1990^{76}$. Aquí resultó clave la reforma fiscal impulsada por Fernández Ordóńez en 1977, ya que aumentó la capacidad recaudatoria de la Seguridad Social ${ }^{77}$.

La mayor parte del sistema de financiación se canalizó durante estos años a través de la Tesorería de la Seguridad Social, que transfería los recursos necesarios al INSALUD, al Instituto Social de la Marina y a las mutuas patronales de accidentes de trabajo para que prestasen la asistencia sanitaria encomendada. Por tanto, existía una dicotomía en el proceso de financiación, pues participaban dos ministerios diferentes, lo que complicaba el proceso ${ }^{78}$. A su vez, el presupuesto del INSALUD se desgaja también desde 1981, año en que comienza el proceso de transferencias sanitarias a las comunidades autónomas con la gestión sanitaria transferida. Por último, el Estado realiza una transferencia a las mutuas de funcionarios (MUFACE, MUGEJU, ISFAS), entidades que permiten completar la asistencia sanitaria pública. En conjunto, el gasto en salud como porcentaje del PIB se incrementó lentamente en Espańa durante estos ańos: 1980: 3,97 \%; 1985: 4,06 \%; 1990: 4,78 \% y 1995: 5,08 \%79. Este modesto crecimiento porcentual ha ido acompañado de una permanente insuficiencia presupuestaria que ha derivado en la conocida deuda sanitaria.

En el año 1983 se intentó poner orden a esta situación ya de por sí insostenible. El director general del INSALUD, Francesc Raventós, denunciaba que la falta de datos hacía imposible cuantificar las plantillas y las deudas contraídas con los proveedores. Además, una buena parte de los mil centros o servicios concertados (hospitales, servicios de hemodiálisis, ambulancias...) carecían de contrato y el resto lo tenía pendiente de revisión desde años atrás ${ }^{80}$. Raventós ponía sus esperanzas de solución en la aprobación de la reforma sanitaria y la aprobación de una ley de administración pública. Mientras tanto, concentró sus esfuerzos en la puesta en marcha de un control estricto del gasto sanitario. Desde mediados de los años ochenta los números del INSALUD empezaron a estar más claros, aunque esto no solucionó el problema de la deuda. La financiación de la sanidad española centrará buena parte de los debates sanitarios

76 Información obtenida de Pons y Vilar (2014): cuadro 4.3.

77 Pérez Jiménez (2000): 254. Sobre la reforma fiscal de la democracia, puede consultarse Albi (1996).

78 Muñoz et al. (1997): 247.

79 Información obtenida de EUROSTAT, accesible en: https:/goo.gl/4usQ43 (consultado el 8 de junio de 2017).

80 «El nuevo año del Insalud», El País, 30-1-1984. 
políticos en las siguientes legislaturas y exigirá varias operaciones de saneamiento para garantizar el funcionamiento del sistema sanitario público.

En tercer lugar, uno de los principales logros de la LGS consistió en dibujar un marco general organizativo y competencial necesario para la acción pública sanitaria del Estado descentralizado tratando de hacer compatible la autonomía de cada territorio con un sistema sanitario único basado en la igualdad y la solidaridad. De este modo la LGS puso las bases para construir una red única de centros con financiación pública. Dentro de este marco, resultó fundamental la integración de todas las estructuras y servicios públicos al servicio de la salud dentro del Sistema Nacional de Salud tanto de la Administración del Estado como de las comunidades autónomas ${ }^{81}$.

Un cuarto aspecto clave, incluido en la LGS, se refiere a la gestión de los recursos sanitarios. Recordemos que en los países que adoptaron SNS, la provisión de los servicios sanitarios se organizó de manera directa, mientras que en los de SS se realizó de manera indirecta. Así, en el primer grupo de países encontramos en teoría una integración entre el financiador y el proveedor de los servicios sanitarios. Sin embargo, en la práctica, la mayoría de países bajo un SNS han adoptado una gestión mixta de los servicios, aunque con un claro predominio de la propiedad pública en los hospitales y privada en la atención primaria (con la excepción de España, Grecia y Portugal, que en estos años mantenían también la propiedad pública en la atención primaria).

¿Qué modelo de gestión implantó la LGS? La normativa consagraba como regla general la gestión directa de la administración con sus propios medios y plantilla. De este modo, el Estado no solo establecía las reglas del servicio y las financiaba, sino que se encargaba además de la provisión y suministro de las prestaciones sanitarias ${ }^{82}$. No obstante, esta regla general contenía tres excepciones: primera, la mayoría de las prestaciones farmacéuticas no se producían y suministraban directamente, sino a través de farmacias privadas; segunda, los beneficiarios de las mutualidades administrativas que financian la asistencia sanitaria (MUFACE, ISFAS y MUGEJU), pero no han desarrollado una infraestructura propia, sino que contratan los servicios con empresas

81 LGS, título III, art. 42. El proceso de transferencias sanitarias a las comunidades autónomas se inició con Cataluńa en 1981; siguió con Andalucía (1984), País Vasco y Valencia (1988); Navarra y Galicia (1991), Canarias (1994) y se cerró con el traspaso sanitario del resto de comunidades en 2002 . Una vez terminado este proceso se aprobó la Ley de Cohesión y Calidad del SNS en junio de 2003 para lograr el equilibrio entre descentralización y coordinación, Ley 16/2003, BOE 29/05/2003, núm. 128, pp. 20.567-20.589.

Muñoz et al. (1997): 272-273. 
privadas y públicas, entre las que los beneficiarios pueden elegir, siempre que exista un convenio con el SNS; por último, la LGS establecía la posibilidad de firmar convenios o conciertos con el sector privado para la prestación de servicios sanitarios o complementarios: «Las Administraciones Públicas Sanitarias, en el ámbito de sus respectivas competencias, podrán establecer conciertos para la prestación de servicios sanitarios con medios ajenos a ellas. A tales efectos, las distintas Administraciones Públicas tendrán en cuenta, con carácter previo, la utilización óptima de sus recursos sanitarios propios» (art. 90.1). No obstante, «a los efectos de establecimiento de conciertos, las Administraciones Públicas darán prioridad, cuando existan análogas condiciones de eficacia, calidad y costes, a los establecimientos, centros y servicios sanitarios de los que sean titulares entidades que tengan carácter no lucrativo" (art. 90.2). Los centros sanitarios susceptibles de ser concertados por «las Administraciones Públicas Sanitarias deberán ser previamente homologados por aquéllas, de acuerdo con un protocolo definido por la Administración competente, que podrá ser revisado periódicamente» (art. 90.5).

En conjunto, la LGS impulsada por Ernest Lluch en el ocaso de la primera legislatura socialista supuso un paso clave para establecer las bases del modelo sanitario español, pero su elaboración y aprobación consumió los primeros cuatro años de gobierno del PSOE, por lo que dejó muchos flecos pendientes en cuanto a su aplicación práctica. Por otro lado, su excesiva generalidad exigió una aplicación progresiva de sus contenidos a través de medidas complementarias. La prensa de la época señalaba al final de la primera legislatura socialista, en la primavera de 1986, que «la esperada reforma global de la sanidad sigue siendo una promesa por cumplir..., pues los proyectos de reforma hospitalaria y de asistencia sanitaria primaria apenas han comenzado a aplicarse» ${ }^{83}$. Como consecuencia, sus sucesores en el Ministerio de Sanidad tenían por delante una doble tarea: poner en práctica la reforma sanitaria y desarrollar el proceso de transferencia de las competencias sanitarias a las comunidades autónomas en un marco de restricciones presupuestarias y duros ajustes de cara al proceso de integración de España en la Unión Europea. Quedaba todavía mucho camino por recorrer.

\section{CONCLUSIONES: LA AGENDA PENDIENTE TREINTA AÑOS DESPUÉS}

La LGS supuso un enorme progreso en la configuración de un modelo sanitario propio de un Estado democrático moderno, al estilo de otros países

83 «El cambio apenas ha tocado la estructura sanitaria», El Pais, 10-6-1986. 
europeos, aunque solo estableció directrices básicas y objetivos generales en materia sanitaria que exigían el desarrollo de posteriores reformas más concretas. No obstante, cabe destacar que treinta años después de su adopción, y con alternancia política en los Gobiernos, las bases de la LGS no se han modificado. El debate sanitario continuó en el centro de la arena política en la década siguiente, en torno a tres cuestiones básicas: las listas de espera en la sanidad, la necesidad de contener el gasto y ganar eficiencia y el modelo de gestión sanitaria, temas muy relacionados entre sí. Dentro de este contexto, la suficiencia financiera se convirtió en uno de los objetivos principales de las administraciones sanitarias en un marco europeo que iniciaba los recortes en bienestar y exigía a España ajustes en las cuentas públicas de cara a su integración en Europa.

A principios de la década de 1990, se produjeron tres victorias a pequeña escala: la ampliación de la cobertura sanitaria hasta conseguir casi la protección universal; la progresiva financiación del gasto del Estado vía impuestos y en detrimento de las cotizaciones sociales; y la ley del medicamento, que pretendía contribuir a la mayor eficacia y calidad de los fármacos. En paralelo, las conclusiones del Informe Abril (1991), encargado por el ministro de sanidad (García Vargas) a una comisión de expertos en un clima de debate social sobre la privatización de la sanidad en España, cayeron como una bomba en el ámbito político y la opinión pública. El informe (cuya elaboración costó más de cien millones de pesetas) proponía en el fondo modelos de gestión privada en la sanidad pública, recortes de gasto, pagos por algunos servicios sanitarios y privatización de algunas actividades en un marco donde el déficit sanitario alcanzaba alrededor de 700000 millones de pesetas ${ }^{84}$. Lejos de conseguir el consenso, el informe provocó una falla más grande entre partidos políticos, profesionales y ciudadanía. Finalmente, quedó guardado en un cajón.

En las últimas décadas del siglo xx los diferentes Gobiernos trataron de frenar el gasto, sobre todo en farmacia (control de recetas, acuerdos con la industria, impulso de genéricos), con los conocidos popularmente como «medicamentazos». Por otro lado, comenzaron a introducirse otros modelos de gestión hospitalaria que diluyeron la línea fronteriza entre la sanidad privada y pública a través de las fundaciones sanitarias, la firma de conciertos y la gestión privada de instalaciones sanitarias públicas.

El balance histórico presenta luces y sombras. De un lado, el seguro obligatorio de salud llegó tarde a España y bajo una dictadura, con una cobertura escasa, carencia de infraestructuras y una financiación deficiente basada en cotizaciones sociales. El joven modelo público sanitario de la democracia se

84 «El Informe Abril sugiere un sistema de financiación de la sanidad que implica la desaparición del Insalud», El País, 6-7-1991. 
vio asolado antes de configurarse por la falta de consenso político y la falta de recursos que obligaban a recortar gastos y servicios. La aprobación de la LGS en los ańos ochenta pareció un punto de inflexión en este camino de incertidumbres, aunque no logró el consenso político. Hoy en día el modelo sanitario público español se enfrenta a un futuro incierto en un marco de crisis, recortes, privatizaciones de la gestión y retroceso del estado de bienestar. De otro lado, y a pesar de todos estos problemas, el sistema sanitario español ocupa las primeras posiciones de los rankings mundiales y sus principales indicadores se sitúan entre los mejores del mundo. El largo camino recorrido y las dificultades históricas superadas invitan a ser moderadamente optimistas, aunque muchos aspectos puedan ser mejorables.

\section{Bibliografía}

Albi, E. (1996). La reforma fiscal de la democracia. Hacienda Pública Española, número extraordinario, 281-295.

Barbado, F. J. y Powell, C. (dir.). (2011). El PSOE y la reforma sanitaria, ¿ un nuevo modelo? En Congreso Internacional Historia de la Época Socialista: 1982-1996. Disponible en: https://goo.gl/Fpb6xQ.

Carr, R. (2009). España: 1808-2008. Barcelona: Ariel.

Comín, F. (1996). Las formas históricas del Estado de bienestar: el caso español. En B. Álvarez-Miranda (ed.). Dilemas del estado de bienestar (pp. 29-58). Madrid: Fundación Argentaria.

- (2010). Los seguros sociales y el Estado de bienestar en el siglo xx. En J. Pons y J. Silvestre (coords.). Los orígenes del Estado de bienestar en España, 1900-1945: los seguros de accidente, vejez, desempleo y enfermedad (pp. 17-50). Zaragoza: Prensas Universitarias de Zaragoza.

- (2015). La deuda pública: el bálsamo financiero del régimen de Franco (1939-1975). Revista de Historia Industrial, 57 (1), 173-210.

Elola, J. (2001). Politica sanitaria española. Madrid: Díaz de Santos.

Esping-Andersen, G. (1990). The Three Worlds of Welfare capitalism. Cambridge: Polity Press.

- (ed.). (1996). Welfare States in Transition. National Adaptations in Global Economies. London: Sage.

Ferrera, M. (1996). The 'Southern model' of welfare in social Europe. Journal of European SocialPolicy, 6(1), 17-37.Disponibleen:https://doi.org/10.1177/095892879600600102.

Francia, J. M. (1997). 25 años de Reforma sanitaria. De la transición al Gobierno del Partido Popular 1970-1995. Salamanca: Hespérides.

Gallego, F. (2008). El mito de la transición. La crisis del franquismo y los origenes de la democracia (1973-1977). Barcelona: Crítica.

García Delgado, J. L., Fusi, J. P. y Sánchez Ron, J. M. (2008). España y Europa. En J. Fontana y R. Villares (dirs.). Historia de España (vol. 11). Barcelona: Crítica-Marcial Pons. 
Giménez, M. C. (2011). La transición hacia la reforma sanitaria: la Ley General de Sanidad (1986). En Congreso Internacional Historia de la Época Socialista: 1982-1996. Disponible en: https://goo.gl/r5bcwc.

Guillén, A. M. (2000). La construcción politica del sistema sanitario español: de la postguerra a la democracia. Madrid: Exlibris.

- (2006). Los sistemas sanitarios públicos europeos y el sistema sanitario público español. En Actas de las Jornadas sobre "La Sanidad Pública en España: reflexiones» (pp. 31-46). Oviedo: Consejo Económico y Social del Principado de Asturias.

Jurado, E. (1993). Crónica de la transición sanitaria en España (1977-1992). Del discreto encanto de la reforma prometida al Informe Abril. Madrid: E. Jurado.

Linz, J. J., Montero, J. R. y Ruiz, A. M. (2005). Elecciones y política. En A. Carreras y X. Tafunell (coords). Estadísticas Históricas de España. Siglos XIX-XX (vol. III) (pp. 1027-1154). Madrid: Fundación BBVA.

Medeiros, J. y Schwierz, C. (2015). Efficiency estimates of health care systems. European Economy. Economic Papers, 549. Luxembourg: Publications Office of the European Union. Disponible en: https://goo.gl/5WNQN2.

Muñoz, J. L., García, J. L. y González, L. (dir.). (1997). Las estructuras del Estado de bienestar. Derecho, economía y sociedad en España. Madrid: Civitas.

Pérez Jiménez, R. (2000). Políticas sanitarias y desigualdades en España. En J. Adelantado (coord.). Cambios en el Estado de bienestar (pp. 251-284). Barcelona: Icaria.

Pons, J. y Vilar, M. (2014). El seguro de salud privado y público en España. Su análisis en perspectiva histórica. Zaragoza: Prensas Universitarias de Zaragoza.

Sevilla, F. (2006). La universalización de la atención sanitaria. Sistema Nacional de Saludy Seguridad Social. Madrid: Ministerio de Trabajo y Asuntos Sociales.

Temes, J. L. y Gil, J. (1996). El Sistema Nacional de Salud. Madrid: McGraw-Hill Interamericana.

Vallejo, R. (2003). Estado y economía en la España democrática, 1975-1999. Historia y Politica: Ideas, Procesos y Movimientos Sociales, 9, 159-184.

Vilar, M. (2004). La ruptura posbélica a través del comportamiento de los salarios industriales: nueva evidencia cuantitativa (1908-1963). Revista de Historia Industrial, 25, 81-126. 